\title{
NAC-1 Is a Brain POZ/BTB Protein That Can Prevent Cocaine-Induced Sensitization in the Rat
}

\author{
Scott A. Mackler, ${ }^{1-5}$ Laxminarayana Korutla, ${ }^{2,4}$ Xian-Yuan Cha, ${ }^{1,3}$ Mark J. Koebbe, ${ }^{2,4}$ Keith M. Fournier, ${ }^{5}$ \\ M. Scott Bowers, ${ }^{6}$ and Peter W. Kalivas ${ }^{6}$
}

Departments of ${ }^{1}$ Medicine and 2 Psychiatry, Philadelphia Veterans Administration Medical Center, and Departments of ${ }^{3}$ Medicine, 4 Psychiatry, and 5Pharmacology, University of Pennsylvania School of Medicine, Philadelphia, Pennsylvania 19104, and ${ }^{6}$ Department of Physiology and Neuroscience, Medical University of South Carolina, Charleston, South Carolina 29425

Levels of the mRNA NAC-1 are increased in the rat forebrain weeks after cocaine exposure. This long-term neuroadaptation occurs during the expression of behavioral sensitization, a model of psychostimulant-induced paranoia. NAC-1, the protein encoded by this cocaine-regulated mRNA, contains a Pox virus and zinc finger/bric-a-brac tramtrack broad complex (POZ/BTB) motif, which mediates interactions among several transcriptional regulators. The present studies demonstrate that NAC-1 acts as a transcription factor. NAC-1 was localized to the nucleus of neurons in the brain. Transfection of NAC-1 in cell culture repressed transcription of a reporter gene. NAC-1 was also able to affect the actions of other POZ/BTB proteins in mammalian two-hybrid studies; these interactions required the presence of the POZ/BTB domain. However, NAC-1 appears to be a unique POZ/BTB transcriptional regulator because it does not contain any zinc finger regions found in these other DNA-binding proteins. Adenoviral-mediated overexpression of NAC-1 protein in the rat nucleus accumbens prevented the development but not the expression of behavioral sensitization produced by repeated administration of cocaine. Thus, NAC-1 may modify the longterm behaviors of psychostimulant abuse by regulating gene transcription in the mammalian brain.

Key words: behavioral sensitization; cocaine; DNA; neuron; POZ protein; rat; transcription factor
Members of the Pox virus and zinc finger/bric-a-brac tramtrack broad complex (POZ/BTB) family share a protein-protein interaction motif in the $\mathrm{N}$ terminal that mediates homodimer and heterodimer formation (Bardwell and Treisman, 1994; Albagli et al., 1995). Many of these proteins contain zinc finger regions that bind DNA, allowing the proteins to regulate gene transcription. In humans the POZ/BTB proteins BCL- 6 and promyelocytic leukemia zinc finger act as powerful oncoproteins when altered by genomic translocations (Chen et al., 1993; Kerckaert et al., 1993). Several POZ/BTB mRNAs are present in the mammalian CNS at levels much higher than those observed in peripheral tissues (Bardwell and Treisman, 1994; Albagli et al., 1995), but the functional significance of this unequal expression is unknown. NAC-1 is a member of the POZ/BTB family, and $N A C-1$ mRNA levels remained elevated weeks after cocaine use (Cha et al., 1997). This increase was restricted to the nucleus accumbens, a forebrain structure critical for the manifestation of many addictive behaviors (Koob, 1996). Reduction of NAC-1 expression after antisense oligonucleotide injection into the nucleus accumbens augmented the acute locomotor responses to systemic cocaine (Kalivas et al., 1999). This finding suggested that the induction of $N A C-1$ by repeated cocaine administration may be a homeostatic response to limit the cocaine-induced behaviors. Because repeated cocaine administration results in the emergence of maladaptive behaviors indicative of addiction, such as drug craving and paranoia (O'Brien,

Received March 21, 2000; revised May 16, 2000; accepted May 22, 2000.

This work was supported by a Department of Veterans Affairs Merit Review grant and National Institutes of Health Grants KDA00199, RO1DA11809, RO1DA03906, and T32DA0724109. We thank Julie Blendy, Thomas R. Kleyman, and Charles P. O'Brien for helpful discussions, Vivian Bardwell (ZID and BCL-6 constructs) and Mitch Lazar (Gal4-NCoR and Gal4-luciferase) for plasmid DNAs, Rong Wen for help with adenoviral work, Diane M. Lewis for technical assistance with Figure $1 \mathrm{~A}$, and the Philadelphia Veterans Administration Medical Center Medical Media Service.

Correspondence should be addressed to Dr. Scott A. Mackler, Medical Research Service (151C), Philadelphia Veterans Administration Medical Center, Philadelphia, PA 19104. E-mail: smackler@mail.med.upenn.edu.

Copyright (C) 2000 Society for Neuroscience $0270-6474 / 00 / 206210-08 \$ 15.00 / 0$
1995), it is possible that the induction of NAC-1 may limit the emergence of these negative behavioral neuroadaptations.

Experiments in the present study characterize NAC-1 as a transcription factor similar to other nuclear POZ/BTB proteins except for the absence of a known DNA-binding domain. In addition, the interactions between NAC-1 and the behavioral effects of cocaine were further evaluated using adenoviral transfer of the NAC-1 cDNA into cells in the nucleus accumbens. Because of the previous observation that microinjection of antisense oligonucleotides directed against $N A C-1$ augmented the locomotor response to an acute systemic injection of cocaine (Kalivas et al., 1999), it was hypothesized that overexpression of this transcription factor would inhibit the motor stimulant effect of cocaine. Moreover, repeated cocaine administration in rodents results in behavioral sensitization that can be operationally defined as the progressive augmentation in cocaine-induced motor stimulation (Post and Rose, 1976; White and Kalivas, 1998). Behavioral sensitization is thought to model the development of paranoia in psychostimulant addicts (Segal and Schuckit, 1983), and it was hypothesized that the overexpression of NAC-1 would inhibit the development of behavioral sensitization to repeated cocaine injections.

\section{MATERIALS AND METHODS}

Generation of a polyclonal anti-NAC-1 antibody and immunohistochemistry. The peptide CTNDPRRKPLDSR, located within the non-POZ/BTB domain of NAC-1, was synthesized and used to immunize a rabbit (the cysteine was added to couple the peptide to limpet hemocyanin). Enzymelinked immunoadsorbent assays demonstrated the presence of antibodies that recognized this antigenic peptide after three immunizations. Radiolabeled NAC-1 protein was synthesized in vitro (Promega, Madison, WI) with the addition of $\left[{ }^{35} \mathrm{~S}\right]$ methionine and incubated with the serum, followed by immunoprecipitation of any antigen-antibody complexes with protein A-agarose. For immunohistochemistry, rats were killed with an overdose of pentobarbital $(100 \mathrm{mg} / \mathrm{kg}$, i.p.), and the brains were perfused via intracardiac inf usion of $4 \%$ paraformaldehyde in PBS. The brains were removed and stored overnight in the paraformaldehyde, and coronal tissue sections ( 15 or $50 \mu \mathrm{m}$ thick) were made for immunohistochemical evaluation of the location of NAC-1 protein. A soluble peroxidase-antiperoxidase complex (Aldrich, Milwaukee, WI) was used along with nickel enhancement of a 3,3-diaminobenzidine color reaction. The primary antibodies were diluted 1:500 in PBS containing 1\% normal goat serum 
A
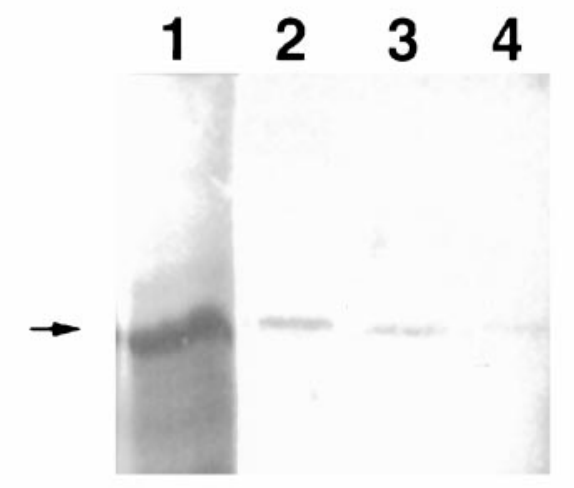

B
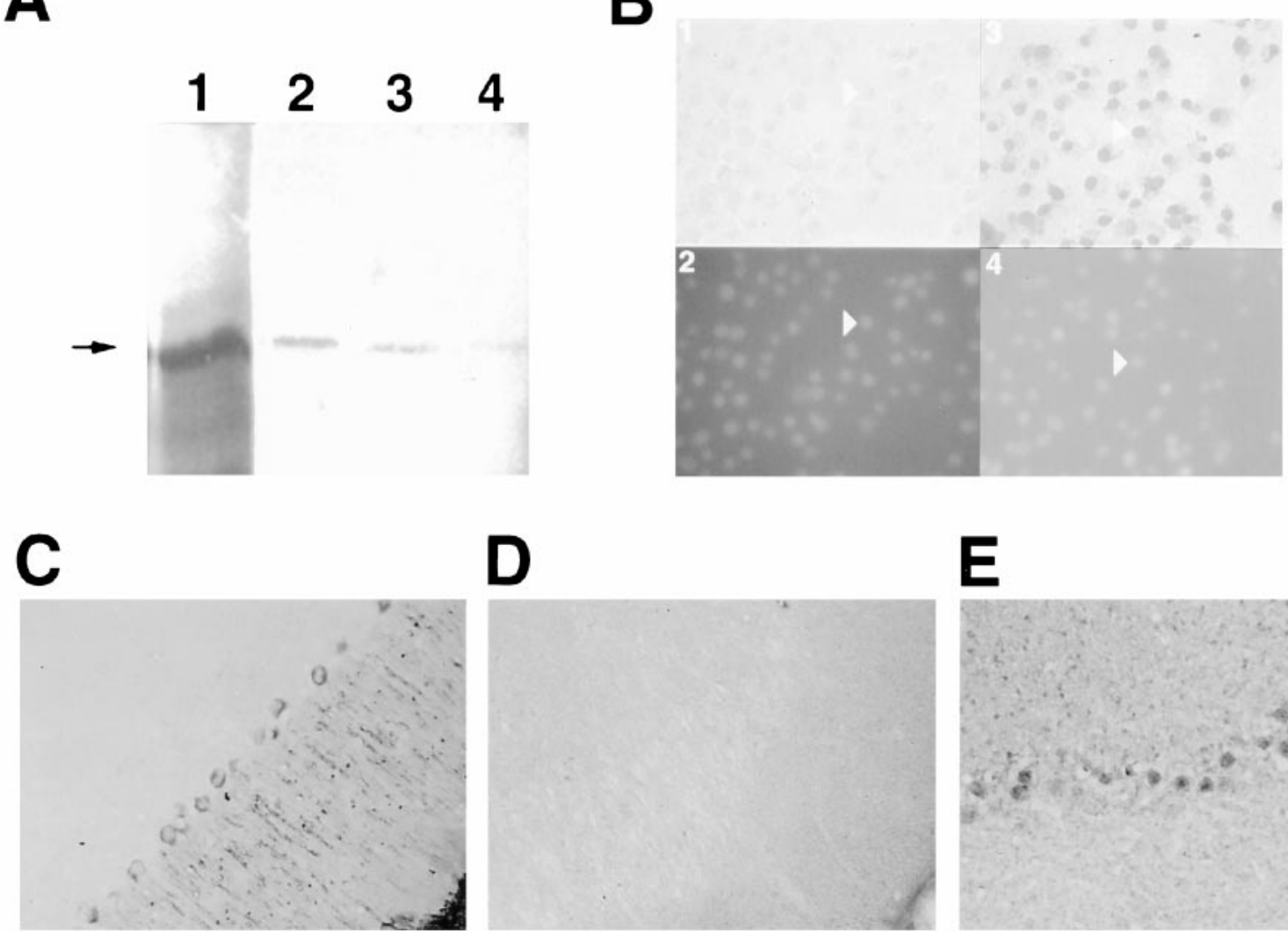

D


Figure 1. NAC-1 is localized to the cell nucleus. A, A single band (arrow) of the correct size was present after in vitro translation of NAC-1 (lane 1), and $50 \%$ of this amount was used as input for each immunoprecipitation. A similar-sized band was present after immunoprecipitation with a 1:500 dilution of antibody (lane 2); less protein was immunoprecipitated using a 1:1000 dilution of antibody (lane 3). Coincubation of a 1:500 dilution of antibody and free peptide markedly decreased immunoprecipitation (lane 4). B, Cos-7 cells were examined with either preimmune serum (panel 1) or anti-NAC-1 polyclonal antibody (panel 3). The area of NAC-1 staining overlaps with each cell nucleus, as determined by counterstaining with the DNA-binding dye 4'6-diamidino-2-phenylindole (DAPI; panels 2, 4); one cell on each side is indicated for comparison (arrowheads). C, The distribution of the cytoplasmic protein $\alpha$-tubulin in rat cerebellar Purkinje cells is restricted to the periphery of the cell body and also the large dendrites that project to the surface. $D$, A section processed with preimmune serum does not reveal any staining. This and the other tissue sections are oriented with the pial surface to the bottom and right. E, NAC-1 protein staining is restricted to the central region of Purkinje cells and is not present in the large dendrites. Smaller cells that also appear to contain NAC-1 protein are in the granular cell layer.

and $0.25 \%$ Triton X-100 $\alpha$-Tubulin was detected with a monoclonal antibody (Amersham, Arlington Heights, IL). To verify gene transfer in the adenovirus study (see below), immunocytochemistry for NAC-1 was conducted as described above. Green fluorescent protein (GFP) or Escherichia coli $\beta$-galactosidase (lacZ) cDNAs inserted in the control adenovirus were used as reporter genes. GFP was visualized using a fluorescence excitor filter in the UV range $(330-380 \mathrm{~nm})$ and a barrier filter at $420 \mathrm{~nm}$. For $\beta$-galactosidase detection, sections were incubated for $1 \mathrm{hr}$ at $37^{\circ} \mathrm{C}$ with the chromogenic 5-bromo-4-chloro-3-indolyl- $\beta$-D-galactopyranoside substrate (Navarro et al., 1999). In addition, to evaluate the viral gene transfer into glia, sections were labeled for glial expression of glial fibrillary acidic protein (GFAP; 1:800; Sigma, St. Louis, MO) or microglial expression of integrin $\alpha \mathrm{M}$ (1:1200; Chemicon International). The GFAP-indocarbocyanine (-Cy3) primary antibody was incubated with tissue for $8 \mathrm{hr}$ in the presence of $1 \%$ normal goat serum plus $0.25 \%$ Triton X-100, washed in PBS, and mounted onto slides. After incubation for $2 \mathrm{hr}$ with the integrin $\alpha \mathrm{M}$ primary antisera, the tissue was washed and incubated for $2 \mathrm{hr}$ with a goat anti-mouse-Cy3 secondary antibody (1:1000; Chemicon International). Double labeling was determined by switching between the Cy3 (590 $\mathrm{nm})$ and the GFP ( $420 \mathrm{~nm}$ ) barrier filter at a magnification of $200 \times$. Cell counts were made in two $500 \times 700 \mu \mathrm{m}$ square regions of high GFP labeling for each of seven animals injected with a recombinant adenovirus containing GFP (Ad-GFP) 1 week before killing for immunocytochemistry (see above). In addition to immunocytochemistry, some tissue sections were stained using a cresyl violet Nissl procedure to permit additional evaluation of neurotoxicity.

Transfections, mammalian two-hybrid experiments, and transcription assays. Plasmids were cotransfected by calcium/phosphate precipitation into H293T (see Fig. $2 B, C$ ) or Cos-7 (see Fig. $2 D$ ) cells along with a plasmid encoding luciferase downstream of multiple Gal4 elements and a second plasmid encoding $\beta$-galactosidase (Chang et al., 1996). After $\sim 48$ $\mathrm{hr}$, the cells were lysed, and both luciferase and $\beta$-galactosidase activities were measured. Western blots were performed to ensure that all Gal4 fusion proteins were synthesized. In the mammalian two-hybrid experiments, either Gal4-zinc interaction domain (-ZID; see Fig. 2C) or Gal4-
BCL-6 (see Fig. 2D) represented the acceptor protein, and either NAC-1 or NAC-1 lacking the POZ/BTB domain (dNAC-1) represented the donor protein. Luciferase values were normalized to the $\beta$-galactosidase results to adjust for transcription efficiency. Each set of transfections was performed a minimum of three times. A Student's $t$ test with a correction for multiple groups was used for statistical analysis.

Electrophoretic mobility shift assays. Gel shift assays were completed as described previously (Bardwell and Treisman, 1994). Briefly, proteins were synthesized in vitro using a coupled transcription-translation system (Promega), and the success of all reactions was determined with SDS-PAGE analysis. The NAC-1 cDNA constructs were inserted in the same T7 Plink vector used for the ZID cDNA constructs. The DNA probe was labeled by PCR amplification with $\left[\alpha^{-32} \mathrm{P}\right] \mathrm{dCTP}$ and allowed to bind to $\sim 20 \%$ of the synthesized protein for $20 \mathrm{~min}$ at room temperature. This mixture then underwent electrophoresis in a nondenaturing, $6 \%$ polyacrylamide gel.

Construction of NAC-1 and control adenovirus. The open reading frame of NAC-1 was subcloned into the shuttle vector pCA14 (Microbix). This vector contains the cytomegalovirus (CMV) promoter. Recombinant adenoviruses were formed by cotransfection of this plasmid with replicationdeficient adenovirus type 5 DNA into $\mathrm{H} 293$ cells. A recombinant adenovirus containing the NAC-1 cDNA (Ad-NAC) was isolated, confirmed by restriction mapping and PCR amplification, and used for large-scale purification of live virus. By the use of the same protocol, Ad-GFP and a recombinant adenovirus containing $\beta$-galactosidase (Ad-lac) were constructed, purified, and used as controls for the transfer of a foreign gene in the in vivo transfection studies.

Overexpression of $N A C-1$ protein in the rat brain and behavioral measures. Male Sprague Dawley rats (250-300 gm; Harlan Laboratories) were anesthetized using a combination of ketamine and xylazine, and bilateral microinjection needles were stereotaxically placed into the nucleus accumbens [anteroposterior, $9.0 \mathrm{~mm}$; mediolateral, $2.0 \mathrm{~mm}$; and dorsoventral, $0.0 \mathrm{~mm}$ (Pellegrino et al., 1979)]. The microinjection of adenovirus $\left(10^{7}\right.$ plaque-forming units/side) was made over $60 \mathrm{sec}$ in a volume of $0.7 \mu \mathrm{l}$. Bilateral microinjection of adenovirus encoding lacZ or GFP served as the control. In addition, in one group of animals Ad-NAC was microinjected 
A

Figure 2. NAC-1 can alter transcription of the Gal4-luciferase reporter gene. $A$, The different proteins used in transient transfections are diagrammed. Control levels of transcription represent transfection of only the Gal4 DNA-binding domain (Gal 4) with the Gal4-luciferase reporter plasmid. All other transfected plasmids encoded Gal4 fusions except for NAC-1 and dNAC- 1 in $C$ and $D . B$, Both the $\mathrm{POZ/BTB}$ and non-POZ/BTB regions of NAC-1 repressed transcription to the same extent as did full-length NAC-1. The nuclear hormone corepressor NCoR as a Gal4 fusion protein was used as a separate measure of repression in this system (Wong and Privalsky, 1998). C, ZID only repressed transcription when full-length NAC-1 was present. $D$, NAC-1 enhanced repression by BCL-6, also an interaction that required a POZ/BTB domain in NAC $-1 .{ }^{*} p<0.01$ versus control; $* * p<0.01$ versus BCL -6 or BCL -6 and dNAC-1. $P O Z_{N}, P O Z / B T B$ domain in NAC-1; $4 x Z n$, zinc fingers in ZID.


B



D

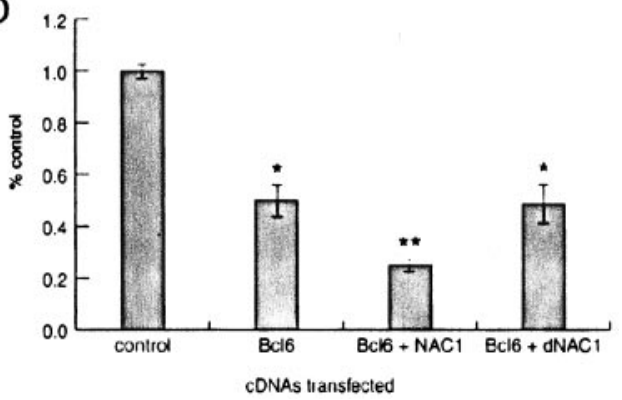

into the striatum dorsal to the injection site in the nucleus accumbens (anteroposterior, $9.0 \mathrm{~mm}$; mediolateral, $2.0 \mathrm{~mm}$; and dorsoventral, $2.5 \mathrm{~mm})$.

Motor activity was monitored in a photocell apparatus (Omnitech Instruments, Columbus, $\mathrm{OH}$ ). The photocell cages have been described in detail elsewhere (Kalivas et al., 1988), and photocell beam breaks were quantified to estimate three motor behaviors. Locomotor behavior was estimated by measuring the distance traveled (determined by quantifying the breaking of adjacent photocell beams), rearing behavior was estimated by the breaking of eight photocell beams located at $8 \mathrm{~cm}$ above the cage floor, and stereotyped behavior was estimated by quantifying repeated breaking of the same photocell beam. Five to seven days after virus was administered into the nucleus accumbens or striatum, the animals were adapted to the photocell apparatus for $1 \mathrm{hr}$. The next day all rats were injected with cocaine $(15 \mathrm{mg} / \mathrm{kg}$, i.p. $)$ after a $1 \mathrm{hr}$ adaptation. This procedure was repeated for the next $6 \mathrm{~d}$. Rats were then allowed to remain in the home cage for $20 \mathrm{~d}$. Twenty-one days after the last cocaine injection, all rats were returned to the photocell cage and after a 60 min adaptation period were injected with cocaine $(15 \mathrm{mg} / \mathrm{kg}$, i.p.). Separate animals were injected with saline $(1.0 \mathrm{ml} / \mathrm{kg}$, i.p. $)$, caffeine $(10 \mathrm{mg} / \mathrm{kg}$, i.p. $)$, or morphine $(3 \mathrm{mg} / \mathrm{kg}$, i.p.) in random order separated by a $3 \mathrm{~d}$ intertrial interval. The motor responses to these acute treatments were monitored using a photocell apparatus as described above. A separate experiment was designed to evaluate the effect of Ad-NAC in the nucleus accumbens on the expression of behavioral sensitization. Animals were administered daily cocaine $(15 \mathrm{mg} / \mathrm{kg}$, i.p., for $7 \mathrm{~d})$, and 1 week after the last injection Ad-NAC or Ad-lac was bilaterally injected into the nucleus accumbens as described above. Two weeks later (day 28) the rats were placed in a photocell cage, and motor activity was monitored as described above.

Statistical analysis of motor responses was conducted using a one- or two-way ANOVA, and a least significant difference test was used for post hoc comparisons between treatment groups (Milliken and Johnson, 1984). Immunohistochemistry was performed as described above to confirm the site and extent of NAC-1 overexpression. Animal use was in compliance with institutional and National Institutes of Health guidelines.

\section{RESULTS}

\section{Antibody characterization and cellular localization of NAC-1}

The specificity of the generated polyclonal antibody was verified by immunoprecipitating NAC-1 made by in vitro translation, and an excess of free peptide significantly reduced this immunoprecipitation (Fig. $1 A$ ). The antibody did not immunoprecipitate the related family member ZID. Figure $1 B$ shows that endogenous NAC-1 expression in Cos-7 cells was detected only in the nucleus because the staining directly overlapped that observed when a DNAbinding dye was used. NAC-1 exhibited a similar pattern of staining in multiple neurons in the rat brain, including cerebellar Purkinje cells (Fig. $1 C-E$ ). The central pattern of staining was complementary to the distribution of the cytoplasmic protein $\alpha$-tubulin. The heterogeneous appearance of NAC-1 staining in brain cells may reflect the speckled distribution observed under confocal microscopy for other POZ/BTB proteins (Bardwell and Treisman, 1994; Albagli et al., 1995). The nuclear localization of NAC-1 is consistent with a function in regulating gene transcription.

\section{The effects of NAC-1 on transcription}

The POZ/BTB domain present in some other proteins can autonomously alter gene transcription (Deweindt et al., 1995; Chang et al., 1996; Kaplan and Calame, 1997). The ability of NAC-1 to either enhance or repress transcription was tested in cultured cells using Gal4 DNA-binding domain fusion proteins (Fig. $2 A$ ) and a Gal4-luciferase reporter gene. Figure $2 B$ reveals that both the $\mathrm{POZ/BTB}$ and non-POZ/BTB domains of NAC-1 produced an $\sim 5$-fold repression of Gal4-luciferase reporter transcription. This is in comparison with the nuclear hormone corepressor NCoR (Hong et al., 1997), which under the same conditions repressed transcription $\sim 10$-fold. A plasmid encoding either full-length NAC-1 or dNAC-1 (both proteins without the Gal4 DNA-binding domain) was cotransfected with plasmids encoding the POZ/BTB proteins BCL-6 (Kerckaert et al., 1993) or ZID (Bardwell and Treisman, 1994) as Gal4 fusion proteins (Fig. 2A). In these mammalian two-hybrid studies, NAC-1 induced (Fig. 2C; ZID) or enhanced (Fig. $2 D$; BCL-6) repression by these CNS proteins. The in vivo interactions required the presence of the NAC-1 POZ/BTB domain because the effects were not present after cotransfection with dNAC-1. These results using transfected cDNAs show that NAC-1 alone is able to repress transcription (Fig. $2 B$ ) and also mediate the actions of other brain POZ/BTB proteins in a POZ/ BTB domain-dependent manner (Fig. 2C,D).

\section{NAC-1 interacts with the binding of the POZ/BTB protein ZID to the ZID DNA element}

The ability of a POZ/BTB protein's zinc finger to bind to its specific DNA element is altered by protein dimerization via the POZ/BTB domain. This dimerization may inhibit DNA binding in vitro via steric hindrance on the DNA-binding domains (Bard- 


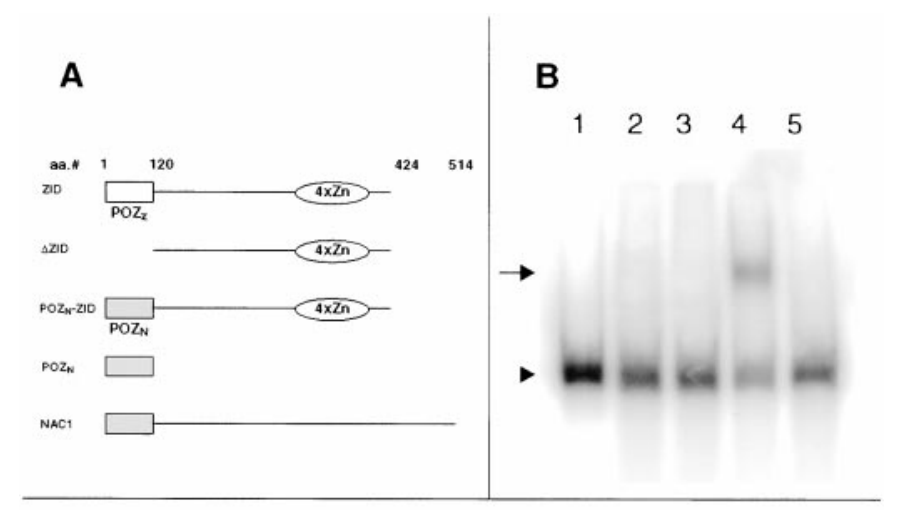

C

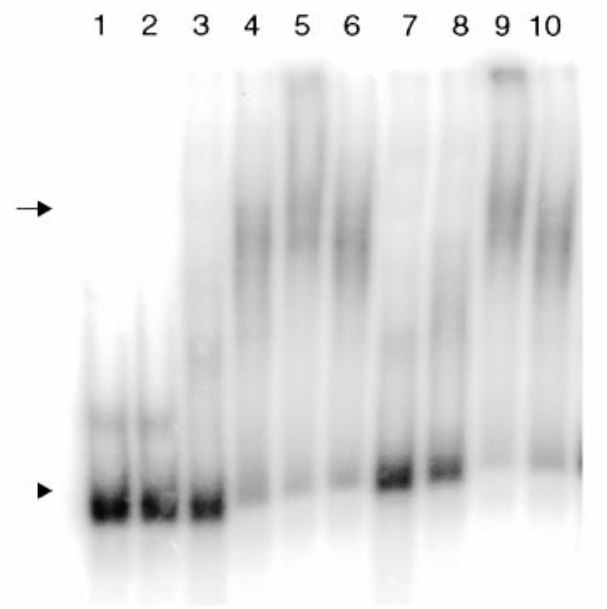

Figure 3. NAC-1 contains a functional POZ/BTB domain. $A$, The different in vitro-translated proteins used in the gel shifts are shown. $P O Z_{Z}$ represents the POZ domain in ZID; $P O Z_{N}$ represents the first 120 amino acids in NAC-1. $B$, Incubation of the radiolabeled ZID DNA element (arrowhead) with bovine serum albumin revealed no effect on the mobility of the probe (lane 1). Incubation of the same probe with $\mathrm{POZ}_{\mathrm{N}}$ (lane 2), a fusion protein of $\mathrm{POZ}_{\mathrm{N}}$ and the ZID zinc fingers (lane 3), or full-length ZID (lane 5) also did not result in a shift. Coincubation of $\mathrm{POZ}_{\mathrm{N}}$ with the $\mathrm{POZ}_{\mathrm{N}}$-ZID zinc finger fusion protein did lead to retardation of the probe (lane 4; arrow; this result is similar to coincubation of $\mathrm{POZ}_{\mathrm{N}}$ with ZID). $C$, Mobility of the ZID DNA element (lane 1; arrowhead) is the same after incubation with albumin (lane 2) or the total protein extract from the striatum of the rat brain (lane 3). The ZID DNA probe does shift after coincubation of striatal protein and $\mathrm{POZ}_{\mathrm{N}}$ (lane 4; arrow) and increases in amount with higher doses of $\mathrm{POZ}_{\mathrm{N}}$ (lanes 5, 6 ). Similar findings occurred with substitution of the protein extract from the cerebellum (lanes 7-10). The broad bands from these shifts presumably reflect complexes of multiple proteins.

well and Treisman, 1994). In contrast, dimerization is thought to enhance the binding of POZ/BTB proteins to genomic DNA in vivo (Katsani et al., 1999). Experiments examined whether the $\mathrm{POZ} / \mathrm{BTB}$ consensus sequence present in NAC-1 could alter the binding of ZID protein to the ZID DNA element. Different in vitro-translated proteins were allowed to bind to the consensus DNA sequence for ZID and used in electrophoretic mobility shift assays. Decreased mobility of the DNA probe (represented by an upward shift) indicates protein binding to the DNA. As expected, the free POZ/BTB portion of NAC-1 by itself did not affect the mobility of the ZID DNA consensus sequence (Fig. 3B, lane 2). A chimeric protein consisting of the NAC-1 POZ/BTB domain fused to the $\mathrm{ZID}$ zinc finger region (Fig. $3 B$, lane 3 ; $\mathrm{POZ}_{\mathrm{N}}-\mathrm{ZID}$ ) also did not bind to the ZID DNA probe, a result similar to that observed with full-length ZID (Bardwell and Treisman, 1994). However, addition of an excess of the NAC-1 POZ/BTB domain "rescued" the ability of this $\mathrm{POZ}_{\mathrm{N}}-\mathrm{ZID}$ fusion protein to bind to the ZID DNA probe (Fig. 3B, lane 4). A shift was also observed after incubation of either the truncated form of ZID that lacks its


Figure 4. NAC-1 overexpression prevents the development of behavioral sensitization. Ad-NAC or control injections of Ad-lac $(n=9)$ or Ad-GFP $(n=4)$ were administered into the nucleus accumbens 1 week before beginning daily injections of cocaine $(15 \mathrm{mg} / \mathrm{kg}$, i.p.) for $7 \mathrm{~d}$ (days 1-7). Three weeks after the last daily cocaine administration (day 28) the rats received another injection of cocaine $(15 \mathrm{mg} / \mathrm{kg}$, i.p.). Motor activity was monitored using a photocell apparatus. Top, The effect of repeated cocaine on different components of cocaine-induced behavioral hyperactivity is shown. Two-way ANOVAs revealed that behavioral sensitization occurred in the distance traveled and rearing only in the AD-lac/Ad-GFP group and that no sensitization of stereotypy was elicited in either treatment group. The significant $F$ scores were as follows: distance traveled, virus $F_{(1,21)}=$ $5.70, p=0.027$, and day $F_{(2.42)}=3.56, p=0.037$; rearing, day $F_{(2,42)} \stackrel{(1,21)}{=} 5.04$, $p=0.011$, and interaction $F_{(2,42)}=3.74, p=0.032$. Bottom, The time course of the locomotor-stimulant response (distance traveled) in response to cocaine is shown. Two-way ANOVAs with repeated measures over time revealed that the motor-stimulant effect of cocaine was greater on days 7 and 28 compared with that on day 1 only in the Ad-lac/Ad-GFP group. Ad-NAC, day $F_{(2,37)}=1.36, p=0.270$, time $F_{(17.629)}=48.08, p<0.001$, and interaction $F_{(34,629)}=0.19, p>0.999$. Ad-lac/Åd-GFP, day $F_{(2,31)}=2.06$, $p=0.145$, time $F_{(17,527)}=70.06, p<0.001$, and interaction $F_{(34,527)}=2.27$, $p<0.001$. $* p<0.05$, comparing day 1 with days 7 and 28 using a least significant difference post hoc comparison (Milliken and Johnson, 1984). Open squares, Day 1; filled squares, Day 7; half-filled squares, Day 28.

POZ/BTB domain or a combination of an excess of the free NAC-1 POZ/BTB domain and full-length ZID with the ZID DNA probe (data not shown). These results show that the NAC-1 POZ/BTB domain functions in a manner similar to that of the ZID POZ/BTB domain (Bardwell and Treisman, 1994) and is able in vitro when fused to the ZID zinc finger regions to prevent protein binding to the ZID DNA element. This protein interaction presumably involves dimerization.

The interaction between the POZ/BTB domains of ZID and NAC -1 raised the possibility that NAC -1 may help to regulate ZID-binding activity in the mammalian brain. The ability of an excess of the NAC-1 POZ/BTB domain to unmask the binding of full-length ZID to the ZID DNA element was used. Incubation of the total protein extract from different brain regions resulted in a shift of the ZID DNA consensus sequence only after addition of the free NAC-1 POZ/BTB domain (Fig. 3C). Incubation of brain extract with full-length NAC-1 or NAC-1 without the POZ/BTB domain did not result in a shift of the ZID DNA probe. The shifts demonstrated a dose-dependent effect, and the resulting broad bands suggest that multiple proteins are involved. These data demonstrate that there is protein(s) present in the mammalian brain that is capable of binding to the ZID DNA element in vitro after interacting with NAC-1's POZ/BTB domain.

\section{Overexpression of NAC-1 in the rat brain}

Figure 4 shows that the rats pretreated with Ad-lac or Ad-GFP demonstrated behavioral sensitization at days 7 and 28 after the 
Table 1. Lack of effect by Ad-NAC administration into the nucleus accumbens on the acute motor response to novelty and psychostimulants

\begin{tabular}{llcc} 
Drug & $\begin{array}{l}\text { Dose } \\
(\mathrm{mg} / \mathrm{kg}, \mathrm{i.p.})\end{array}$ & $\begin{array}{l}\text { Ad-NAC } \\
n=8\end{array}$ & $\begin{array}{l}\text { Ad-lac } \\
n=7\end{array}$ \\
\hline Novelty & - & $8636 \pm 854$ & $8570 \pm 358$ \\
Saline & - & $1766 \pm 303$ & $1816 \pm 329$ \\
Morphine & 3.0 & $5774 \pm 1175$ & $6579 \pm 1102$ \\
Caffeine & 10.0 & $21245 \pm 944$ & $20944 \pm 318$
\end{tabular}

All data are shown as the mean ( \pm SEM) distance-traveled photocell counts over 120 min after drug injection, except in rats exposed to novelty in which the data are the distance traveled accumulated over $60 \mathrm{~min}$ after placement into the open field. An unpaired, two-tailed Student's $t$ test revealed no significant difference between the Ad-NAC and Ad-lac pretreatment groups.

first cocaine injection. The sensitized motor response was revealed in both locomotor activity (distance traveled) and rearing behavior, but not in stereotyped behavior. The time course analysis revealed that significant behavioral sensitization occurred during the first 30 min after cocaine administration. In contrast, the Ad-NAC-pretreated rats did not exhibit sensitization or any significant difference between treated days in any of the measured behaviors. Comparing the first day of cocaine administration between the virus treatment groups reveals that the acute motor response to cocaine did not differ. Similarly, both groups of rats demonstrated equivalent motor responses to a novel environment (the first exposure to the photocell chamber) and to the acute intraperitoneal administration of saline, morphine, or caffeine (Table 1). Thus, the blockade of behavioral sensitization by Ad-NAC pretreatment did not result from a general inhibitory effect on motor activity.

In agreement with the literature (for review, Peltekian et al., 1997), the adenoviral-mediated overexpression of transgenes endures for at least the $35 \mathrm{~d}$ of the behavioral experiment (see Fig. 6). Thus, it is possible that rather than disrupting the development of behavioral sensitization by the daily cocaine treatment regimen, NAC-1 overexpression may have interfered with the expression of the behavioral sensitization elicited by cocaine on day 28 after the 3 week withdrawal period. Figure 5 reveals that the effect of NAC-1 overexpression on behavioral sensitization was selective for the development and not the expression of sensitization because animals microinjected with Ad-NAC 1 week after repeated cocaine administration demonstrated behavioral sensitization at 3 weeks of withdrawal. Behavioral augmentation was observed in both AdNAC- and Ad-GFP-treated groups in the distance traveled and stereotypy but not in rearing behavior. Similar to the selectivity of the nucleus accumbens as a site where cocaine upregulated $N A C-1$ mRNA (Cha et al., 1997), when Ad-NAC was administered into the striatum, repeated cocaine administration continued to elicit sensitization in the measure of distance traveled (Fig. 5).

Immunohistochemistry revealed the presence of substantial cellular overexpression of NAC-1 in subjects injected with the AdNAC (Fig. 6A). Figure $6 B$ shows a similar distribution of cells infected with control Ad-GFP. The overexpression of NAC-1, lacZ, and GFP was typically in the ventral half of the nucleus accumbens and bounded by the anterior commissure and the olfactory tubercle. Thus, transfected cells appeared in both the ventral core and ventrolateral shell compartments. High-power micrographs indicate some morphological differences in cellular overexpression of NAC-1. Figure $6 A^{\prime}$ shows cells with dense nuclear staining and clustered cytoplasmic immune labeling, whereas Figure $6 A^{\prime \prime}$ shows cells resembling the constitutive expression pattern (see Fig. 1) with labeling restricted to the nucleus. The tissue in Figure 6 was obtained from animals at $40 \mathrm{~d}$ after microinjection of virus, showing that robust adenoviral-mediated NAC-1 expression was present for the duration of the experiment. Modest neurotoxicity at the injection site was occasionally observed that was approximately spherical in shape and ranged from 0 to $300 \mu \mathrm{m}$ in diameter. This toxicity was not present in all animals and occurred


Figure 5. NAC-1 did not affect the expression of behavioral sensitization when overexpressed in the accumbens or the development of sensitization when overexpressed in the striatum. Top, The effect of Ad-NAC on the expression of behavioral sensitization was evaluated by administering AdNAC or Ad-GFP into the nucleus accumbens 1 week after the last daily injection of cocaine and 2 weeks later giving a cocaine injection (day 28). The data were evaluated using a two-way ANOVA with repeated measures over days. A significant effect of the day of cocaine treatment was measured for the distance traveled $\left[F_{(1,10)}=8.08 ; p=0.014\right]$ and stereotypy $\left[F_{(1,10)}=\right.$ $10.04 ; p=0.010]$, whereas no effect of virus or interaction between virus and day was found in rearing behavior. Bottom, Ad-NAC was administered into the dorsal striatum 5-7 d before administering the first injection of 1 week of daily cocaine, and cocaine was readministered after 3 weeks of withdrawal (day 28). A one-way ANOVA with repeated measures was conducted, and a significant effect was measured for the distance traveled $\left[F_{(2,20)}=5.28 ; p=0.023\right]$ but not for rearing or stereotypy. ${ }^{*} p<0.05$, comparing day 1 with days 7 and 28 using a least significant difference post hoc comparison (Milliken and Johnson, 1984).

equally in animals injected with Ad-lac, Ad-GFP, and Ad-NAC. Figure $6 C$ shows a Nissl-stained tissue section after microinjection of Ad-NAC and reveals the maximum extent of neurotoxicity observed at the tip of the injection cannula. The neurotoxicity is very restricted; adjacent to the immediate injection site in the region containing an abundance of transfected cells, there is no neurotoxicity, and cell morphology appears normal.

Some cells were found to be double-labeled for GFP and the astroglial marker GFAP at day 7 after microinjection of Ad-GFP (Fig. 7). A cell count of seven brains revealed that $46.0 \pm 6.3 \%$ (149 of 316 cells total) of the GFP-containing cells were double-labeled for GFAP. In contrast, few microglia or macrophages were present at the virus injection site, and only two cells were found to be double-labeled for GFP and integrin $\alpha \mathrm{M}$ (data not shown).

\section{DISCUSSION}

\section{NAC-1 acts as a transcription factor}

This report demonstrates that the cocaine-regulated gene NAC-1 encodes a transcription factor. NAC-1 is localized to the cell nucleus and is able to repress transcription in the context of a foreign gene. NAC-1 is unique in the family of mammalian POZ/ BTB transcriptional regulators because it does not contain a typical zinc finger structure (Mackay and Crossley, 1998). Some POZ/ BTB proteins interact with other nuclear proteins that lack DNAbinding domains, including the nuclear hormone corepressors SMRT and NCoR (Hong et al., 1997; Wong and Privalsky, 1998). NAC-1 may thus alter transcription by either binding directly to DNA (efforts to date have not isolated a target DNA site for NAC-1) or interacting as a cofactor with other proteins that regulate transcription.

NAC-1 required its $\mathrm{POZ/BTB}$ domain to alter repression by two other POZ/BTB proteins, ZID or BCL-6. This domain also affected the binding of proteins containing the ZID zinc fingers to the ZID DNA element in multiple regions of the rat brain, includ- 




Figure 6. Location of gene transfer within the nucleus accumbens. $A$, Lowpower micrograph illustrating Ad-NACmediated overexpression of NAC -1 in the ventral nucleus accumbens. $A^{\prime}$ and $A^{\prime \prime}$ in $A$ refer to the location of the corresponding high-power micrographs. $A^{\prime}$, High-power micrograph showing substantial cytoplasmic localization of NAC-1 in Ad-NACinfected cells. Note the somewhat clustered organization of immune labeling for NAC-1 in the cytoplasm of some cells (arrowheads). $A^{\prime \prime}$, High-power micrograph showing the primarily nuclear location of NAC-1 (arrowheads). B, Distribution of cells from an animal pretreated with AdGFP. C, Low-power micrograph stained with cresyl violet revealing modest neurotoxicity at the injection site. Inset, Highpower micrograph of the boxed area in $C$ showing an abundance of healthy cells in tissue adjacent to the injection site. $a \mathrm{c}$, Anterior commissure. Scale bars: $A-C$ $0.5 \mathrm{~mm} ; A^{\prime}, A^{\prime \prime}, 0.05 \mathrm{~mm}$.
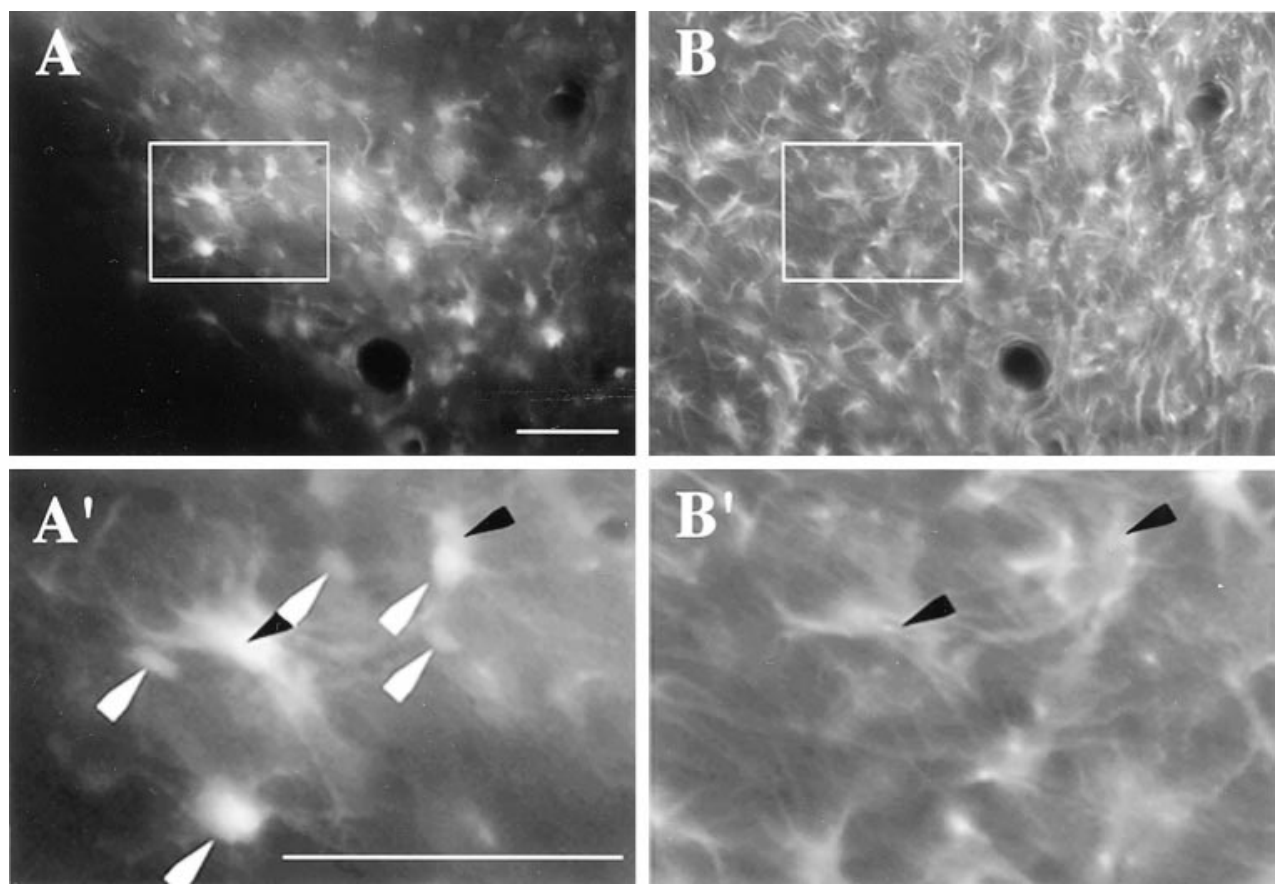

Figure 7. The localization of GFP with glia and neurons. $A$, Expression of GFPcontaining cells in the ventral nucleus accumbens. $B$, Expression of GFAP in the same section shown in $A . A^{\prime}, B^{\prime}$, Higher power micrographs corresponding to the area outlined in $A$ and $B$, respectively. White arrowheads indicate GFP-expressing cells not colocalized with GFAP. Black arrowheads correspond to cells colocalizing GFAP and GFP. Scale bars, $0.1 \mathrm{~mm}$ ing the protein extract from the nucleus accumbens. The NAC-1 POZ/BTB domain almost certainly forms heterodimers and homodimers to mediate these actions, supported by $\mathrm{POZ}_{\mathrm{N}}$ 's migration as a homodimer in electrophoresis (data not shown). Resolution of the POZ/BTB domain structure by crystallization suggests that in vivo obligate homodimers are likely formed, although the homodimers may oligomerize with themselves or other POZ/BTB proteins (Ahmad et al., 1998; Li et al., 1999). The functional ability to form dimers, along with selectivity in these protein-protein interactions, will help to determine the actions of NAC -1 on transcriptional regulation.

\section{NAC-1 is a POZ/BTB protein that can alter a long-term behavioral response to cocaine}

NAC-1 cDNA was first isolated from the brains of rats that had a history of cocaine self-administration (Cha et al., 1997). It was found that $N A C-1$ was selectively upregulated in the nucleus accumbens by repeated cocaine administration and that the overexpression endured for at least 3 weeks after discontinuing daily cocaine administration. The long-term increase in $N A C-1$ levels is unusual when compared with other mRNAs that return to baseline levels within a few days or weeks after drug exposure (Hope et al., 
1992; Moratalla et al., 1996; Berke et al., 1998). This temporal pattern of overexpression is consistent with the enduring behavioral sensitization produced by repeated cocaine administration (Segal and Schuckitt, 1983; White and Kalivas, 1998), indicating that NAC-1 may be a mediator of cocaine-induced behavioral plasticity. The present data reveal that in contrast to being a mediator of behavioral sensitization, the induction of NAC-1 may be a compensatory response to diminish the neuroplastic, longterm impact of cocaine. Rather than augmenting the effect of cocaine, viral-mediated overexpression of NAC-1 in the nucleus accumbens was shown to inhibit the development of behavioral sensitization. This indicates that the constitutive upregulation of NAC-1 by cocaine is functioning to blunt the capacity of the CNS to undergo the cocaine-induced neuroadaptive changes that result in behavioral sensitization. The protective function by NAC-1 is consistent with the previous observation that a reduction in $N A C-1$ expression by microinjecting NAC-1 antisense oligonucleotide into the nucleus accumbens augmented the acute behavioral activation elicited by a systemic injection of cocaine (Kalivas et al., 1999). NAC-1 antisense pretreatment was also found to inhibit selectively the motor response associated with dopamine receptor stimulation in the nucleus accumbens. This indicates that the regulation of gene expression by NAC-1 as a transcription factor may be impacting dopamine receptor signaling and the corresponding changes in gene expression that are associated with acute and repeated cocaine administration (Nestler et al., 1993; White and Kalivas, 1998).

\section{Methodological considerations using adenovirus-mediated gene transfer}

The use of adenovirus to transfer genetic material in vivo suffers from certain shortcomings. The most common caveat to be considered is the induction of an inflammatory response at the site of injection (Akli et al., 1993; Peltekian et al., 1997). Although this occurred in the present study and may have contributed to the slight neurotoxicity observed, by 1 week after injection glial and microglial infiltration of the injection site was not generally present (e.g., see Fig. 7), indicating that the inflammatory response had primarily abated by the time the behavioral experiments began. Another concern is that by using the CMV promoter there is not selective gene transfer into neurons (Le Gal La Salle et al., 1993). Indeed double-labeled cell counts at 1 week after virus administration reveal that nearly $50 \%$ of the cells expressing the reporter gene GFP were glial and not neuronal. Nonetheless, in agreement with other reports (Peltekian et al., 1997), substantial neuronal infection was produced, and studies to date using specific neural promoters reveal a marked reduction in the overall infection rate, although what infection occurs is selective for neurons (Klein et al., 1999; Navarro et al., 1999). Finally, similar to other transgenic techniques, the long duration of overexpression will likely result in adaptations within the infected cells and adjacent neurocircuitry. This is especially true for a protein such as NAC-1 that is capable of modifying gene transcription. How these putative alterations in cell and circuitry function may differ from the overexpression of NAC -1 by repeated cocaine administration cannot be determined in the present study. However, it is important to note that although the constitutive expression of NAC-1 is nuclear, Ad-NAC overexpression resulted in significant protein in the cytoplasm (e.g., compare Figs. 1, 6 $A^{\prime}$ ). Whether this distribution reflects a constitutive cytoplasmic distribution that is below detection by the NAC-1 antibody in untransfected cells or is an artifact of Ad-NACmediated overexpression is not known. Regardless, the magnitude of any Ad-NAC-induced confound will likely be less than that arising from transgene technologies that use constitutive changes in gene expression present for the lifetime of the animal throughout the CNS. The present study was completed within 5 weeks after Ad-NAC administration in adult rats, and the overexpression is produced in a single brain nucleus. Nonetheless, the interpretation of behavioral data obtained using adenoviral-mediated gene transfer will be enhanced by the development of inducible expression systems that minimize the emergence of compensatory adaptations produced by Ad-NAC-induced overexpression that may not occur during cocaine-induced NAC-1 upregulation (Ketz et al., 1999).

\section{Why are POZ/BTB proteins found in neurons?}

Mammalian POZ/BTB transcriptional proteins appear to regulate the cell cycle during hematopoiesis (Ball et al., 1999). POZ/BTB mRNAs are four- to eightfold more abundant in the mammalian brain when compared with peripheral tissues (Bardwell and Treisman, 1994; Albagli et al., 1995), suggesting that POZ/BTB proteins may play an important role in brain function. However, the question of why they may be present in terminally differentiated neurons arises. NAC-1 is the first mammalian POZ/BTB protein implicated in the regulation of a complex behavior. A cascade initiated by the coupling of signal transduction pathways with altered protein expression patterns is thought to induce the neuroadaptations responsible for addictive behaviors (Nestler et al., 1993; Koob, 1996; White and Kalivas, 1998), and this mechanism may underlie a POZ/BTB protein's effect on behavior. Future studies will need to identify what target genes in vivo are regulated by NAC-1 expression and whether this occurs by a direct (DNA-binding) or indirect (transcription cofactor) mechanism. $N A C-1 \mathrm{mRNA}$ is also present in the human brain (data not shown), and characterization of human NAC-1 and its regulated genes may help to initiate new studies into the mechanisms of psychostimulant abuse.

\section{REFERENCES}

Ahmad KF, Engel CK, Prive GG (1998) Crystal structure of the BTB domain from PLZF. Proc Natl Acad Sci USA 95:12123-12128.

Akli S, Caillaud C, Vigne E, Stratford-Perricaudet LD, Poenaru L, Perricaudet M, Kahn A, Pschanski M (1993) Transfer of a foreign gene into the brain using adenovirus vectors. Nat Genet 3:224-228.

Albagli O, Dhordain P, Deweindt C, Lecocq G, Leprince D (1995) The BTB/POZ domain: a new protein-protein interaction motif common to DNA- and actin-binding proteins. Cell Growth Differ 6:1193-1198.

Ball HJ, Melnick A, Shaknovich R, Kohanski RA, Licht JD (1999) The promyelocytic leukemia zinc finger (PLZF) proteins binds DNA in a high molecular weight complex associated with cdc2 kinase. Nucleic Acids Res 27:4106-4113.

Bardwell V, Treisman R (1994) The POZ domain: a conserved proteinprotein interaction motif. Genes Dev 8:1664-1677.

Berke JD, Paletzki RF, Aronson GJ, Hyman SE, Gerfen CR (1998) A complex program of striatal gene expression induced by dopaminergic stimulation. J Neurosci 18:5301-5310.

Cha X-Y, Pierce RC, Kalivas PW, Mackler SA (1997) NAC-1, a rat brain mRNA, is increased in the nucleus accumbens three weeks after chronic cocaine self-administration. J Neurosci 17:6864-6871.

Chang C-C, Ye BH, Chaganti RS, Dalla-Favera R (1996) BCL-6, a POZ/ zinc-finger protein, is a sequence-specific transcriptional repressor. Proc Natl Acad Sci USA 93:6947-6952.

Chen Z, Brand NJ, Chen A, Chen SJ, Tong JH, Wang ZY, Waxman S, Zelent A (1993) Fusion between a novel Kruppel-like zinc finger gene and the retinoic acid receptor alpha locus due to a variant $(\mathrm{t} 11,17)$ translocation associated with acute promyelocytic leukemia. EMBO J 12:1161-1167.

Deweindt C, Albagli O, Bernardin F, Dhordain P, Quief S, Lantoine D, Kerckaert JP, Leprince D (1995) The LAZ3/BCL6 oncogene encodes a sequence-specific transcriptional inhibitor: a novel function for the BTB/ POZ domain as an autonomous repressing domain. Cell Growth Differ 6:1495-1503.

Hong S-H, David G, Wong CW, Dejean A, Privalsky ML (1997) SMRT corepressor interacts with PLZF and with the PML-retinoic acid receptor $\alpha$ and PLZF-RAR $\alpha$ oncoproteins associated with acute promyelocytic leukemia. Proc Natl Acad Sci USA 94:9028-9033.

Hope B, Kosofsky B, Hyman SE, Nestler EJ (1992) Regulation of immediate early gene expression and AP-1 binding in the nucleus accumbens by chronic cocaine. Proc Natl Acad Sci USA 89:5764-5768.

Kalivas PW, Duffy P, DuMars LA, Skinner C (1988) Neurochemical and behavioral effects of acute and daily cocaine. J Pharmacol Exp Ther 245:485-492.

Kalivas PW, Duffy P, Mackler SA (1999) Reduced NAC-1 expression augments the behavioral response to systemic cocaine. Synapse 33:153-159.

Kaplan J, Calame K (1997) The ZiN/POZ domain of ZF5 is required for both transcriptional activation and repression. Nucleic Acids Res 25:1108-1116.

Katsani KR, Hajibagheri MA, Verrijzer CP (1999) Co-operative DNA binding by GAGA transcription factor requires the conserved TB/POZ domain and reorganizes promoter topology. EMBO J 18:698-708.

Kerckaert JP, Deweindt C, Tilly H, Quief S, Lecocq G, Bastard C (1993) LAZ3, a novel zinc-finger gene encoding, is disrupted by recurring 
chromosome 3q27 translocations in human lymphomas. Nat Genet 5:66-70.

Ketz MB, Chen J, Carlezon WA, Whisler K, Gilden L, Beckmann AM, Steffen C, Zhang YJ, Marotti L, Self DW, Tkatch T, Baranauskas B, Surmeier DJ, Nene RL, Duman RS, Picciotto MR, Nestler EJ (1999) Expression of the transcription factor $\mathrm{dFosB}$ in the brain controls sensitivity to cocaine. Nature 401:272-276.

Klein RL, Muir D, King MA, Peel AL, Zolotukhin S, Moller JC, Kruttgen A, Heymach Jr JV, Muzyczka N, Meyer EM (1999) Long-term actions of vector-derived nerve growth factor or brain-derived neurotrophic factor on choline acetyltransferase and Trk receptor levels in the adult rat basal forebrain. Neuroscience 90:815-821.

Koob G (1996) Drug addiction: the yin and yang of hedonic homeostasis. Neuron 16:893-896.

Le Gal La Salle G, Robert JJ, Berrard S, Ridoux V, Stratford-Pericaudet LD, Perricaudet M, Mallet J (1993) An adenovirus vector for gene transfer into neurons and glia in the brain. Science 259:988-990.

Li X, Peng H, Schultz DC, Lopez-Guisa JM, Rauscher III FJ, Marmorstein R (1999) Structure-function studies of the BTB/POZ transcriptiona repression domain from the promyelocytic leukemia zinc finger oncoprotein. Cancer Res 59:5275-5282.

Mackay J, Crossley M (1998) Zinc fingers are sticking together. Trends Biochem Sci 23:1-4.

Milliken G, Johnson D (1984) Analysis of messy data. Designed experiment 1. Belmont, CA: Lifetime Learning.

Moratalla R, Elibol B, Vallejo M, Graybiel AM (1996) Network-level changes in expression of inducible Fos-Jun proteins in the striatum during chronic cocaine treatment and withdrawal. Neuron 17:147-156.
Navarro V, Millecamps S, Geoffroy M-C, Robert J-J, Valin A, Mallet J, Le Gal La Salle G (1999) Efficient gene transfer and long-term expression in neurons using a recombinant adenovirus with a neuron-specific promoter. Gene Ther 6:1884-1982.

Nestler EJ, Hope BT, Widnell KL (1993) Drug addiction: a model for the molecular basis of neural plasticity. Neuron 11:995-1006.

O'Brien CP (1995) Drug addiction and drug abuse. In: The pharmacological basis of therapeutics, 9th Edition (Hardman JG, Limbird LE, eds), pp 557-577. New York: McGraw-Hill

Pellegrino LK, Pellegrino AS, Cushman AJ (1979) A stereotaxic atlas of the rat brain. New York: Plenum.

Peltekian E, Parrish E, Bouchard C, Peschanski M, Lisovoski F (1997) Adenovirus-mediated gene transfer to the brain: methodological assessment. J Neurosci Methods 71:77-84.

Pierce RC, Bell K, Duffy P, Kalivas PW (1996) Repeated cocaine augments excitatory amino acid transmission in the nucleus accumbens only in rats having developed behavioral sensitization. J Neurosci 16:1550-1560.

Post RM, Rose H (1976) Increasing effects of repetitive cocaine administration in the rat. Nature 260:731-732.

Segal DS, Schuckit MA (1983) Animal models of stimulant-induced psychosis. In: Stimulants: neurochemical, behavioral, and clinical perspectives (Creese I, ed), pp 131-167. New York: Raven.

White FJ, Kalivas PW (1998) Neuroadaptations involved in amphetamine and cocaine addiction. Drug Alcohol Depend 51:141-154.

Wong C-W, Privalsky M (1998) Components of the SMRT corepressor complex exhibit distinctive interactions with the POZ domain oncoproteins PLZF, PLZF-RAR $\alpha$, and Bcl-6. J Biol Chem 273:27695-27702. 